\title{
Reliability Modeling and Assessment of Electric Vehicle Motor Using Fault Tree and Fuzzy Petri Nets
}

\author{
Bing Wang ${ }^{1,2}$, Yanping Liang ${ }^{1}$, Chaojun Yang ${ }^{2}$ and Zhibo Sang ${ }^{3}$ \\ ${ }^{1}$ School of Electrical and Electronic Engineering, Harbin University of Science \\ and Technology, Harbin, Heilongjiang 150080, P. R. China \\ ${ }^{2}$ Transportation College, Northeast Forestry University, Harbin, Heilongjiang \\ 150040, P. R. China \\ ${ }^{3}$ Department of Transportation, Jilin Electrical Engineering School, Jilin, 132101 \\ , P. R. China \\ wangbing@nefu.edu.cn
}

\begin{abstract}
Performing reliability analysis of electric vehicle motor has an important impact on its safety. To do so, this paper proposes its reliability modeling and evaluation issues of electric vehicle motor by using fault tree (FT) and fuzzy Petri nets (FPN). Based on the concepts of FT and FPN, a FT based FPN model for reliability analysis is obtained. In addition, the reliability calculation method is introduced and this work designs a hybrid intelligent algorithm integrating fuzzy simulation and $N N$, namely $N N$ based simulation algorithm, to solve it. Finally, taking an electric vehicle motor as an example, its reliability modeling and evaluation issues are analyzed. The results illustrate the proposed models and the effectiveness of proposed algorithms. Moreover, the results reported in this work could be useful for the designers of electric vehicle motor, particularly, in the process of redesigning the electric vehicle motor and scheduling its reliability growth plan.
\end{abstract}

Keywords: Fault tree, Reliability modeling and evaluation, Electric vehicle motor, Uncertainty Theory.

\section{Introduction}

Along with increasing problems of energy and environment, more and more countries develop related policies to handle these changing and disturbing issues, i.e., developing new-energy and low-carbon vehicles, implementing the remanufacturing, reuse and recycling of waste products and performing green transportation technologies [1-5]. Electric vehicle, as an important and green transportation tool, has been widely attracted by more and more researchers [6]. Motor is one of key components of electric vehicle. Its reliability has an important impact on the system safety. Designers have well-recognized the importance of electric vehicle reliability, but to our best knowledge a detailed reliability analysis is still missing. Although the faults have been reduced in the last few years by some measures, it still affects the safety of vehicles, and faults of mechanical system occupy a large proportion of all the faults.

In the present literature, most of the current researches have discussed electric and electronic system issue and reliability prediction analysis of the electric vehicle. For example, Liu et al. present a permanent-magnet synchronous motor drive system of electric vehicles [7]. $\mathrm{Xu}$ et al. discuss driving and control problems of torque for direct-wheel-driven electric vehicle with motors in serial [8]. Quinn et al. present the

*Corresponding author is Bing Wang, E-mail address:liangyanping2010@126.com 
effect of communication architecture on reliability [9]. Zhu et al. present a grey prediction model of motor reliability of electric vehicle [10]. They propose a grey prediction model of electric vehicle motor based on particle swarm optimization [11]. In addition, Zhu et al. discuss the reliability modeling method of solar array based on the fault tree (FT) analysis method [11].

It can be seen from the above literatures that the current research on reliability modeling of electric vehicle motor is limited to FT analysis method. It no doubt that FT analysis has been widely employed as a powerful technique to evaluate the safety and reliability of complex systems by many scholars [12-13]. However, FT analysis has some limitations in reliability analysis. Firstly, in FT analysis, the probabilities of basic events must be known before analysis. Thus based on this assumption, the reliability analysis of the system is only a probability decision-making process and cannot achieve the real-time description of reliability information [14-15]. Secondly, it is not easy for FT analysis to conduct further quantitative analysis automatically due to the lack of effective means of mathematical expression. Thirdly, FT analysis cannot find the dynamic description of fault information of the system precisely and describe the propagation process of fault information.

The Petri nets is one of the mathematical modeling approaches for the description of distributed systems, which consists of places, transitions and directed arcs [16-17]. Many extensions to the Petri nets have been successfully developed and applied in analyzing fault diagnosis, automated manufacturing systems and product disassembly [18-21]. The fuzzy Petri net is a high level one, it has been used to establish models of reconfigurable manufacturing system and network attack due to its better information expression ability and dynamic description performance of process features [22-23]. Although some prior works [22-24] have proposed to use Petri nets to solve reliability issues of nitric acid reactor feed and reconfigurable manufacturing systems, they merely analyze the average failure rate/ life time of system, the real-time probability analysis reliability issues of the system is not yet addressed by using this method. Moreover, for reliability issue of electric vehicle motor, we cannot find reference to handle this issue by fuzzy Petri nets method to the best knowledge of the authors. To do so, this work addresses reliability modeling and evaluation of electric vehicle motor by using fuzzy Petri nets based on fault tree for the first time. Namely, the aim of this work is to find a new way to analyze reliability of electric vehicle motor.

The remainder of this paper is organized as follows: Reliability model and establishment method of FT and fuzzy Petri nets of the mechanical system is given in Section 2. Section 3 presents the reliability analysis method and algorithm. In Section 4, taking an electric vehicle motor as an example, its reliability modeling and evaluation is presented. Section 5 concludes our work and describes some future research issues.

\section{FT based Fuzzy Petri nets Models for Reliability Analysis}

Reliability model is basis and premise for reliability analysis and evaluation, thus we first introduce the concept and establishment process of Fuzzy Petri nets model based on FT for reliability analysis. To easily establish it, the following method is proposed in this work. Namely, the FT model for reliability analysis is established based on its related concept, and then transformation rules of elements of FA to Petri nets are defined. Finally, FT based fuzzy Petri nets model for reliability analysis is established.

\subsection{FT Model for Reliability Analysis}

FT is the most usual model of reliability analysis. Many references describe it in detail [25-26]. In this work, we only present its basic elements and schematic diagram.

1) Basic elements of FT

Usually, FT is composed of a series of events and logic gates. The main events include: 
Top event: it is the most undesirable system failure event and the object of the analysis. It is denoted as

Middle event: it is the sub-system or component failure event and the cause of the tope vent. It is denoted as

Basic event: it is the primary failure event and the cause of the top event or middle events. It is denoted as $\bigcirc$.

The main logic gates include:

Logic OR gate: it indicates that output event occurs if either one of the input events occurs. It is denoted as .

Logic AND gate: it indicates that output event occurs only if all of the input events occur. It is denoted as

There may be many other types of events and logic gates involved in complex system reliability analysis. However, for the sake of concisions, we only list the most commonly used ones here. For other types of events and logic gates, please refer to [25-26].

2) FT model of a mechanical system

Based on the presented basic elements and logical relationship of fault occurrence, the schematic diagram of FT for reliability analysis of a mechanical system is presented next, as shown in Fig. 1.

As shown in Fig. 1., this FT is composed of 1 top event, 2 middle events and 4 basic events. They are T, M1 and M2, and B1, B2, B3 and B4.

\subsection{Fuzzy Petri nets Model for Reliability Analysis}

1) Concept of extended Petri nets

The Petri net is a graphic modeling method, which is widely used in modeling and analyzing discrete event systems such as semiconductor manufacturing, transportation and automated manufacturing systems. A fuzzy Petri nets is a high lever one. It is a type of improved fuzzy Petri nets with fuzzy distribution. Before giving the formal definition, we present the definition of PN introduced by Petri in1962 [27-30].

A PN is a five-tuple $(\mathrm{P}, \mathrm{T}, \mathrm{I}, \mathrm{O}, \mathrm{m})$, where:

$P=\left\{p_{1}, p_{2}, \mathrm{~L}, p_{n}\right\}, \mathrm{n}>0$, and is a finite set of places pictured by circles;

$T=\left\{t_{1}, t_{2}, \cdots, t_{s}\right\}, \mathrm{s}>0$, and is a finite set of transitions pictured by bars, with $P \cup T \neq \Phi, P \cap T=\Phi$

$I: P \times T \rightarrow N$ and is an input function that defines the set of directed arcs from $\mathrm{P}$ to $\mathrm{T}$ where $N=\{0,1,2 \ldots\}$; 


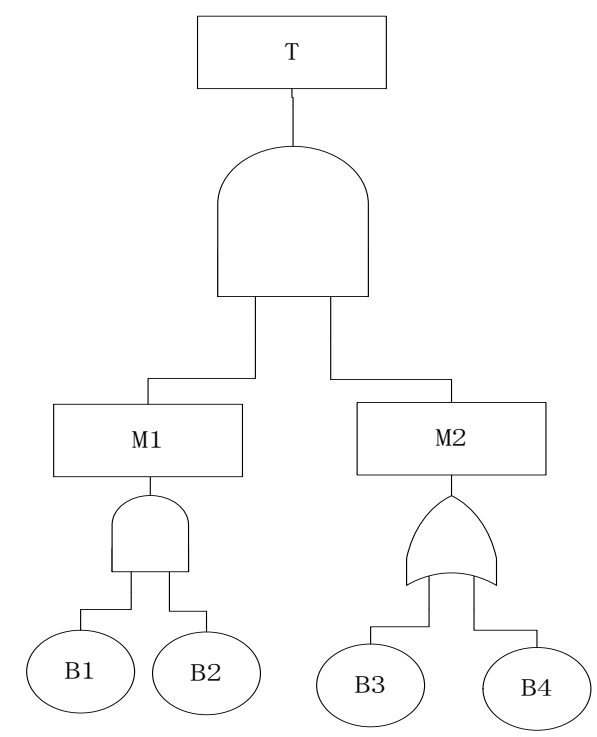

Figure 1. FT model for Reliability Analysis of a Mechanical System

$O: T \times P \rightarrow N$ and is an output function that defines the set of directed arcs from T to $\mathrm{P}$;

$m_{i}: P \rightarrow N$ and is a marking whose ith component represents the number of tokens in the ith place. An initial marking is denoted by $\mathrm{m} 0$. The tokens are pictured by dots.

A simple PN and its elements are shown in Fig. 2. The four-tuple (P, T, I, O) is called a PN structure that defines a directed graph structure. A PN models system dynamics using tokens and their firing rules.

Assume that every transition in a PN is associated with a fuzzy variable delay from the enabling to the firing of the transition, and then this PN is transformed into the fuzzy Petri net, while very transition in a PN is associated with arbitrary distribution fuzzy delay, this PN is called as extended Petri nets. It is defined as follows [31]:

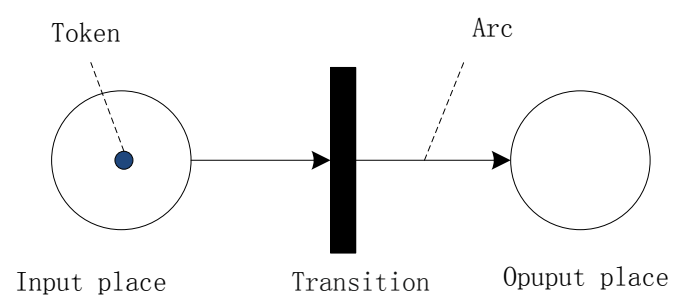

Figure 2. A Simple PN and its Elements

A fuzzy Petri nets is a six-tuple $(\mathrm{P}, \mathrm{T}, \mathrm{I}, \mathrm{O}, \mathrm{m}, \mathrm{H})$, where:

$P=\left\{p_{1}, p_{2}, \cdots, p_{n}\right\}, \mathrm{n}>0$, and is a finite set of places;

$T=\left\{t_{1}, t_{2}, \cdots, t_{s}\right\}, \mathrm{s}>0$, and is a finite set of transitions, with $P \cup T=\Phi$, $P \cap T=\Phi$

$I: P \times T \rightarrow N$ and is an input function that defines the set of directed $\operatorname{arcs}$ from $\mathrm{P}$ to $\mathrm{T}$ where $N=\{0,1,2 \ldots\}$;

$O: T \times P \rightarrow N$ and is an output function that defines the set of directed arcs from $\mathrm{T}$ to $\mathrm{P}$; 
$m_{i}: P \rightarrow N$ and is a marking whose ith component represents the number of tokens in the ith place. An initial marking is denoted by $\mathrm{m} 0$.

$H: T \rightarrow R$, is a vector whose component is a firing time delay with an extended/arbitrary distribution function.

Note that the firing time delay is the life time of corresponding component in fuzzy Petri nets model for reliability analysis.

2) Elements transformation rules of FT to Petri nets

To obtain the extended Petri nets, we introduce the transformation rules of elements of FT to ones of fuzzy Petri nets. Based on different logic relations, the transformation of AND/OR gates of FT to AND/OR transitions of fuzzy Petri nets is shown in Fig. 3.

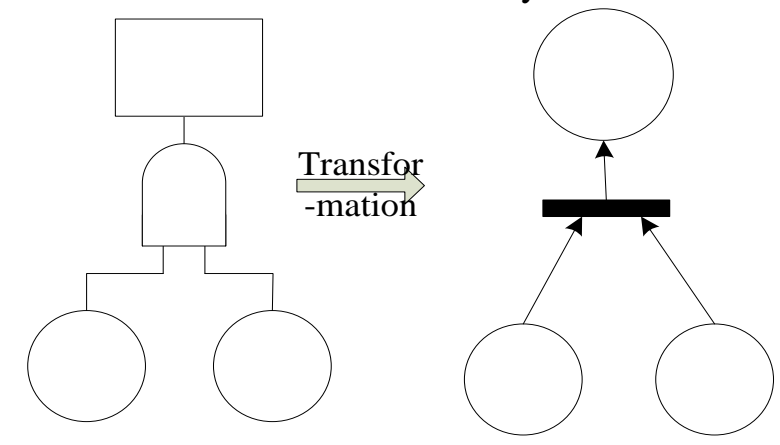

(a) Transformation of AND gate

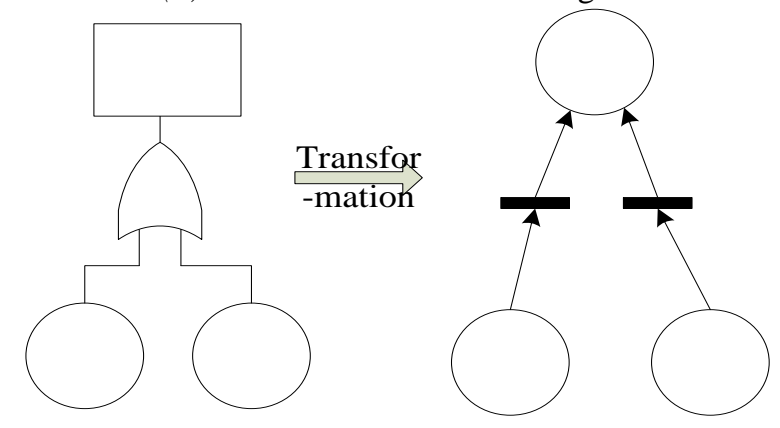

(b) Transformation of OR gate

Figure 3. Transformation of AND/OR Gate

As shown in Fig. 3., the top/middle/basic event, logical gate and logical relation line of FT are transformed to place, transition and arc of extended Petri nets, respectively.

3) The establishment of fuzzy Petri nets based on FT

Based on the FT and elements transformation rules of FT to fuzzy Petri nets, the FT based fuzzy Petri nets model for reliability analysis is obtained, as shown in Fig. 4. 


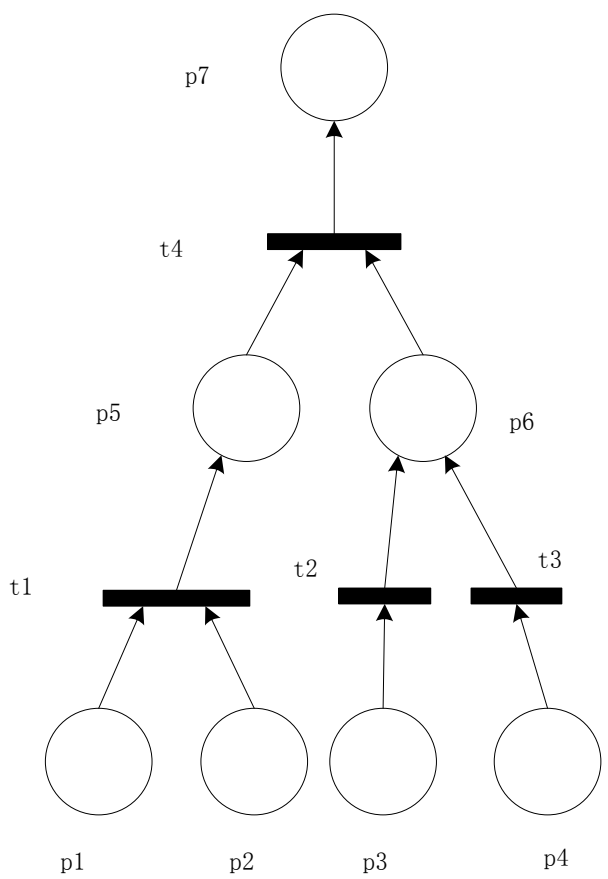

Figure 4. Extended Petri nets Model for Reliability Analysis of a Mechanical System

From Figs. 4 and 1, compared to FT model, we can see that the building FT model need 6 types of elements, i.e., 3 types of events, 2 types of logic gates and a kind of relation line, while the building extended Petri nets model only need 3 types of elements, i.e., place, transition and arc. Namely, the fuzzy Petri nets model is more concise than FT one. In addition, when each transition is associated with its corresponding life distribution function, it can achieve the real time description of reliability analysis. Also, this model can achieve the dynamic delivery and propagation of reliability/fault information due to the introduction of transition and directed arc. Overall, the results denote that using fuzzy Petri nets method to establish the product reliability model is more convenient, concise and effective than FT model.

\section{Method and Algorithm for Reliability Evaluation}

\subsection{Reliability Evaluation Method}

1) Reliability evaluation parameters and calculation method

To easily establish our models, the concept of fuzzy variable, assumptions and parameters are given next.

Concept of fuzzy variable

Fuzzy set theory is introduced by Zadeh (1965), and is well developed and applied in a wide variety of practical problems. In the fuzzy world, there are three important types of measures: possibility, necessity, and credibility (Liu 2004).

Let $\xi$ be a fuzzy variable with membership function ${ }^{\mu}$, and let $\xi$ and $\mathrm{r}$ be real numbers. The possibility, necessity, and credibility of a fuzzy event $\{\xi \leq r\}$ are defined respectively by

$$
\begin{gathered}
\operatorname{Pos}\{\xi \leq r\}=\sup _{t \leq r} \mu(u) \\
\operatorname{Nec}\{\xi \leq r\}=1-\operatorname{Pos}\{\xi>r\}=1-\sup _{t>r} \mu(u)
\end{gathered}
$$




$$
\operatorname{Cr}\{\xi \leq r\}=\frac{1}{2}(\operatorname{Pos}\{\xi \leq r\}+N e c\{\xi \leq r\})
$$

After giving the concept of credibility, the concept of the expected value of a fuzzy variable $t$ can be defined as follows:

$$
E(\xi)=\int_{0}^{+\infty} C r\{\xi \geq r\} d r-\int_{-\infty}^{0} C r\{\xi \leq r\} d r
$$

In addition, to computer system reliability, the life calculation method of AND/OR transition in extended Petri nets is pretended next.

Let a specified system consists of $\mathrm{n}$ components and the life of this $\mathrm{n}$ components be $x_{1}, x_{2}, \cdots$, and $\mathrm{x}_{\mathrm{n}}$, respectively.

For AND transitions, the system life $\mathrm{x}$ is expressed as,

$$
\mathrm{x}=\min \left\{\mathrm{x}_{1}, \mathrm{x}_{2}, \ldots, \mathrm{x}_{\mathrm{n}}\right\}
$$

For OR transitions, the system life $\mathrm{x}$ is expressed as,

$$
\mathrm{x}=\max \left\{\mathrm{x}_{1}, \mathrm{x}_{2}, \ldots, \mathrm{x}_{\mathrm{n}}\right\}
$$

For example, in Fig. 4, let the life of corresponding components of places $p_{1}$ and $p_{2}$ be $\mathrm{x}_{1}$ and $\mathrm{x}_{2}$, respectively, then the life of corresponding system $\mathrm{x}_{5}$ of places $\mathrm{p}_{5}$ is $\mathrm{x}_{5}=\min \left(\mathrm{x}_{1}\right.$, $\mathrm{x}_{2}$ ). Let the life of corresponding components of places $\mathrm{p}_{3}$ and $\mathrm{p}_{4}$ be $\mathrm{x}_{3}$ and $\mathrm{x}_{4}$, respectively, then the life of corresponding system $\mathrm{x} 6$ of places $\mathrm{p}_{6}$ is $\mathrm{x}_{6}=\max \left(\mathrm{x}_{3}, \mathrm{x}_{4}\right)$.

For other systems, its life calculation can be obtained by the integration of AND and OR transition calculation. Such as, a system as shown in Fig. 4 , its system life $x_{7}=\min \left\{x_{5}\right.$, $\left.\mathrm{x}_{6}\right\}=\min \left\{\min \left(\mathrm{x}_{1}, \mathrm{x}_{2}\right), \max \left(\mathrm{x}_{3}, \mathrm{x}_{4}\right)\right\}$.

We know that with the opportunity to represent the credibility measure of fuzzy events more accurately, let's discuss how to calculate the credibility of fuzzy event by fuzzy simulation.

Suppose $\xi=\left(\xi_{1}, \xi_{2}, \cdots, \xi_{n}\right)$ is a vague possibility vector space $(\Theta, p(\Theta), P o s)$, Are uniformly generated from $\Theta$ to $\theta_{k}$, so that $\operatorname{Pos}\left\{\theta_{k}\right\} \geq \varepsilon$, and definitions $v_{k}=\operatorname{Pos}\left\{\theta_{k}\right\}, k=1,2, \cdots N$, where in $\varepsilon$ is a sufficiently small number. Credibility which is $\operatorname{Cr}\{f(\xi) \leq r\}$ can be estimated by the following formula:

$$
L=\frac{1}{2}\left(\max _{1 \leq k \leq N}\left\{v_{k} \mid f\left(\xi\left(\theta_{k}\right)\right) \leq r\right\}+\min _{1 \leq k \leq N}\left\{1-v_{k} \mid f\left(\xi\left(\theta_{k}\right)\right)>r\right\}\right)
$$

\subsection{Algorithm for Reliability Evaluation}

Fuzzy simulation is an effective means to assess and calculate fuzzy and probabilistic functions. It has effectively solved many fuzzy programming problems [32-35]. While neural networks (NN) have been successfully used to solve many complex industrial evaluation and optimization problems due to their strong nonlinear fitting ability [36-38], we propose to use a fuzzy simulation algorithm based on $\mathrm{NN}$ to solve the reliability of the proposed FT based extended Petri nets model.

1) Fuzzy simulation of reliability function

Step 1: $s=\sqrt{u+v}+b$;

Step 2: Randomly generate real numbers $u_{i}$ of the $\varepsilon$ - level sets of fuzzy variables $\xi_{i}$ such that $s=\sqrt{u+v}+b$ and $s=\sqrt{u+v}+b$;

Step 3: Set $s=\sqrt{u+v}+b s=\sqrt{u+v}+b$;

Step 4: Randomly generate $r$ from [a, b];

Step 5: If $s=\sqrt{u+v}+b$, then $s=\sqrt{u+v}+b$;

Step 6: If $s=\sqrt{u+v}+b$, then $s=\sqrt{u+v}+b$;

Step 7: Repeat the fourth to sixth steps for $\mathrm{N}$ times; and 
Step 8: $s=\sqrt{u+v}+b$.

2) Neural networks (NN)

$\mathrm{NN}$ is treated as a nonlinear mapping system consisting of neurons (processing units), which are linked by weighted connections. It usually consists of three layers: input, hidden, and output layers. There is an activation function in the hidden layer. It is defined as the sigmoid function in this paper [36-39].

Firstly, the method to determine the number of neurons of the input, hidden, and output layer is presented as follow.

The number of input neurons of an NN structure is the number of bottom places in extended Petri nets model, namely, the number of basic event in FT model, in other words, the number of input neurons is the number of life distribution of basic fault component of a system in this paper.

The number of output neurons is 1 representing one system life function. In terms of the NN structure, the main problem is to determine the best number of hidden neurons. The number can be infinite in theory, but finite in practice due to two reasons. Too many hidden neurons increase the training time and response time of the trained NN. On the other hand, too few hidden neurons make the NN lack of generalization ability. Therefore, it can usually be determined by the following formula, namely, $s=\sqrt{u+v}+b$, where $u$ and $v$ are the number of input neurons and output neurons, respectively, and $\mathrm{b}$ is a constant from 1 to 10 [40]. Based on it, in terms of a system shown in Fig. 4, $u$ is set to be 4 since this system is composed of 4 bottom places, $v=1$, thus $s$ is a constant from 3 to 12 .

Secondly, backpropagation is the most commonly used method to calculate values for the weight and bias terms of an NN model. In this method, all weights are adjusted according to the calculated error term using a gradient method. Learning in an NN, that is the calculation of the weights of the connections, is achieved by minimizing the error between its output and the actual output over a number of available training data points. In this paper, the error term is controlled by the following Matlab function, namely net.train Param.goal. It denotes the mean squared error between the output of the neural network and the actual output over a number of available training data points.

Thus the NN algorithm is presented as follows:

Step 1: Initialize the number of neurons at the input, hidden, and output layers, and initialize weight vector $\mathrm{w}$.

Step 2: Calculate the output of the hidden layer, the output of output layer and adjust the corresponding weights $\mathrm{w}$.

Step 3: Calculate the error term, namely training performance goal. If it is larger than the given error, go to Step 2, and otherwise, end.

3) NN based simulation algorithm

Based on the presented fuzzy simulation and NN, the steps of the fuzzy evaluation algorithm based on $\mathrm{NN}$ are presented as follows:

Step 1: Initialize the parameters of an NN structure and the number of training data points $K$.

Step 2: Establish an FT based fuzzy Petri nets model for reliability analysis based on fault logic relationships of each component in a system.

Step 3: Based on the relationship among the system life and corresponding each component life in FT based extended Petri nets model, generate the input-output data for $\mathrm{NN}$ training by the fuzzy simulation technology.

Step 4: Train the NN to approximate the uncertain function, namely the transition transmission rule/relation of the system life calculation, and obtain output data of a system life of the NN.

Step 5: Forecast outputs of system life value are obtained by the NN algorithm. 
Step 6: Calculate reliability degree of $\mathrm{R}(\mathrm{x})$ and average life $\mathrm{E}(\mathrm{x})$ by fuzzy simulation and obtained forecast outputs of system life value.

The above algorithm has been implemented in the MATLAB (R2009b) programming language.

\section{Reliability Modeling and Evaluation of Electric Vehicle Motor}

\subsection{Reliability Modeling of Electric Vehicle Motor}

As shown in Fig. 5, it is a schematic graph of electric vehicle motor. It mainly consists of 3 parts, i.e., stator, rotor and axis. According its components and their logical relation of fault occurrence, combined with the presented concept of FT, its FT model is shown in Fig. 6.

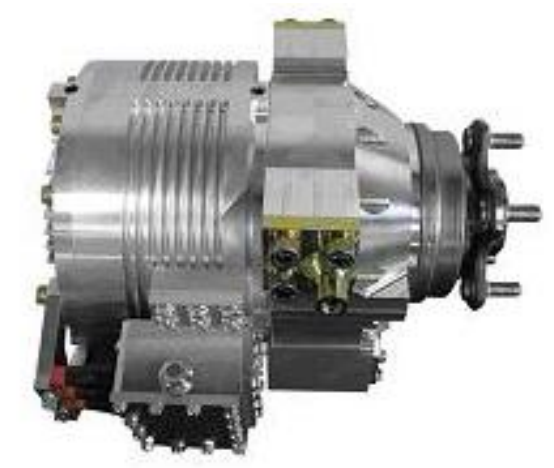

Figure 5. Motor of Electric Vehicle

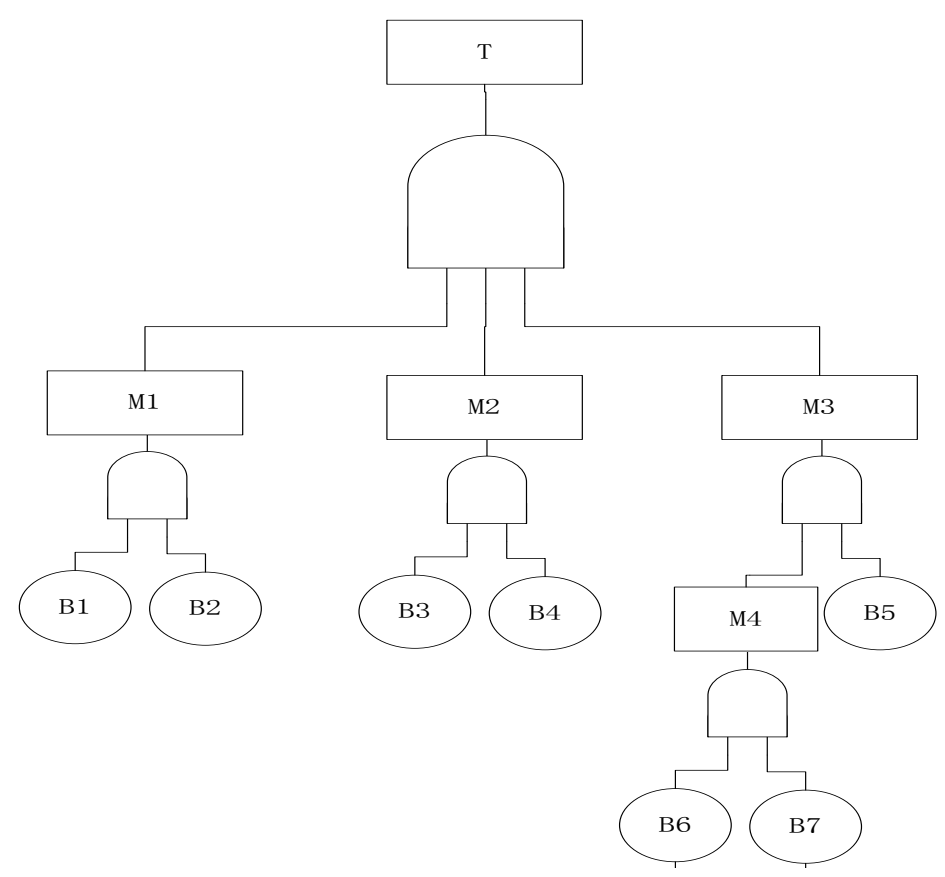

Figure 6. FT Model of Motor of Electric Vehicle

Based on elements transformation rules of FA to fuzzy Petri nets, the FT based fuzzy Petri nets model of motor of electric vehicle is obtained and shown in Fig. 7. 


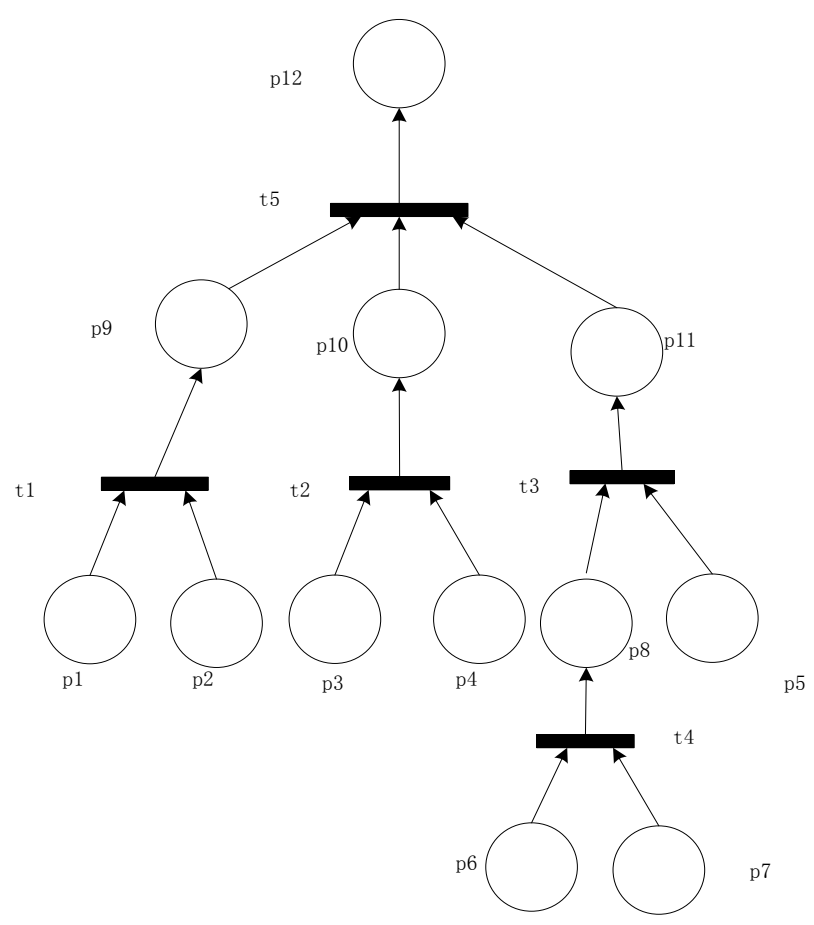

Figure 7. Extended Petri nets Model of Electric Vehicle Motor

In addition, the paraphrase for the transitions and places of FT based fuzzy Petri nets model of electric vehicle motor is listed Tables 1 and 2, respectively. Additionally, the life distribution types and parameters associated with each transition is listed Table 2. Note that Triangle $(\mu, \sigma)$ denote normal distributions, The distribution parameters can be determined by the life test of motor and the unit of life time is hour [41].

Table 1. Events and Paraphrases of Each Place in FT and Extended Petri Nets Models

\begin{tabular}{ccc}
\hline FT & $\begin{array}{c}\text { extended Petri } \\
\text { nets }\end{array}$ & Paraphrase \\
\hline B1 & p1 & Failure of core of the stator \\
B2 & p2 & Failure of winding of the stator \\
B3 & p3 & Failure of core of the rotor \\
B4 & p4 & Failure of winding of the rotor \\
B5 & p5 & Failure of bearing \\
B6 & p6 & Failure of the shaft \\
B7 & p7 & Failure of the shaft assembly \\
M4 & p8 & Failure of the stator \\
M1 & p9 & Failure of the rotor \\
M2 & p10 & Failure of the axis system \\
M3 & p11 & Failure of the motor system \\
T & p12
\end{tabular}


Table 2. Paraphrases of Each Transition in Extended Petri Nets Models

\begin{tabular}{|c|c|c|}
\hline $\begin{array}{l}\text { extended } \\
\text { Petri nets }\end{array}$ & Paraphrase & $\begin{array}{l}\text { Life distribution type } f(x) \text { associated with } \\
\text { transition } t\end{array}$ \\
\hline $\mathrm{t} 1$ & $\begin{array}{l}\text { Faults of core and } \\
\text { winding of the stator } \\
\text { cause fault of stator }\end{array}$ & $\begin{array}{l}\text { Life distribution of core of the stator } x_{1} \text { is } \\
\text { Triangle }(18000,20000,22000) ; \\
\text { Life distribution of winding of the stator } x_{2} \text { is } \\
\text { Triangle }(8000,9000,10000)\end{array}$ \\
\hline $\mathrm{t} 2$ & $\begin{array}{l}\text { Faults of core and } \\
\text { winding of the rotor } \\
\text { cause fault of the rotor }\end{array}$ & $\begin{array}{l}\text { Life distribution of core of the rotor } x_{3} \text { is } \\
\text { Triangle }(16000,18000,20000) ; \\
\text { Life distribution of winding of the rotor } x_{4} \text { is } \\
\text { Triangle }(9000,10000,11000)\end{array}$ \\
\hline $\mathrm{t} 3$ & $\begin{array}{l}\text { Faults of bearing and } \\
\text { shaft assembly cause } \\
\text { fault of the axis system }\end{array}$ & $\begin{array}{c}\text { Life distribution of bearing } x_{5} \text { is } \\
\text { Triangle }(8000,10000,12000) \text {; Life distribution of } \\
\text { shaft assembly is obtained by } x_{6} \text { and } x_{7}\end{array}$ \\
\hline $\mathrm{t} 4$ & $\begin{array}{l}\text { Faults of shaft and bond } \\
\text { cause fault of shaft } \\
\text { assembly }\end{array}$ & $\begin{array}{c}\text { Life distribution of shaft } x_{6} \text { is } \\
\text { Triangle }(16000,18000,20000) \text {; Life distribution of } \\
\text { bond } x_{7} \text { is Triangle }(14000,16000,18000)\end{array}$ \\
\hline $\mathrm{t} 5$ & $\begin{array}{l}\text { Faults of the stator, } \\
\text { rotor and axis system } \\
\text { cause the fault of the } \\
\text { motor system }\end{array}$ & $\begin{array}{l}\text { Life distributions of stator, rotor and axis system } \\
\text { are obtained by calculation of life of constituting } \\
\text { its components }\end{array}$ \\
\hline
\end{tabular}

\subsection{Reliability Evaluation of Electric Vehicle Motor}

The parameters of the $\mathrm{NN}$ based simulation algorithm are set as follows: for FT based extended Petri nets model of electric vehicle motor, since there are 7 bottom place in this model, the number of input neurons is set to be $u=7$,. Thus the number of hidden neurons is 12 by letting $b=12$. The given error term value is 0.000000004 . Based on the fuzzy simulation, 4000 input-output data points are generated and obtained. Moreover, they are separated into two groups: 3000 data points for training and 1000 points for testing. Note that the number of training data points is set to be 4000 due to two reasons. First, too few data points make the solution of models inaccurate. Second, too many points increase the training time and response time of the solution model. Note that the solution accuracy cannot be increased significantly with the number of data points [36-37].

1) The results of average life $E(x)$ of electric vehicle motor

Based on the established FT based extended Petri nets model and the above presented algorithm, after the proposed fuzzy simulation algorithms based on $\mathrm{NN}$ is executed, the average life $\mathrm{E}(\mathrm{x})$ of electric vehicle motor is obtained, i.e., $\mathrm{E}(\mathrm{x})=8895.5$ hours. It denotes that the average use time of this electric vehicle motor is 8895.5 hours.

2) The results of reliability degree $\mathrm{R}(\mathrm{x})$ of electric vehicle motor

Given the different running time $\mathrm{x}$ of the electric vehicle motor, after the proposed fuzzy simulation algorithms based on NN is executed, the corresponding reliability degree $\mathrm{R}(\mathrm{x})$ is obtained, as listed in Table 3 . 
Table 3. Reliability Degree of Electric Vehicle Motor under Different Running Time

\begin{tabular}{cccccc}
\hline$x$ & 8000 & 8200 & 8400 & 8600 & 9000 \\
\hline$R(x)$ & 0.943 & 0.884 & 0.876 & 0.840 & 0.801 \\
\hline
\end{tabular}

As seen in Table 3, for example $\mathrm{R}(8200)=0.884$, it denote that the probability of this motor, which does not occur fault, is 0.871 when it has run for 8200 hours time.

In addition, in order to make future tests and observe the effectiveness of the proposed methods, the predicted outputs of $\mathrm{NN}$ method and their output error between forecast and actual outputs at test data points are shown in Figs. 8 and 9.

From Figs. 8 and 9, the predicted and output results of the proposed algorithm are highly close. It reveals that the proposed algorithm can accurately achieve reliability evaluation of the electric vehicle motor.

\section{Conclusions}

Electric vehicle motor is one of key components in electric vehicle and has a great impact on the vehicle safety, thus it is important to perform its reliability analysis. To

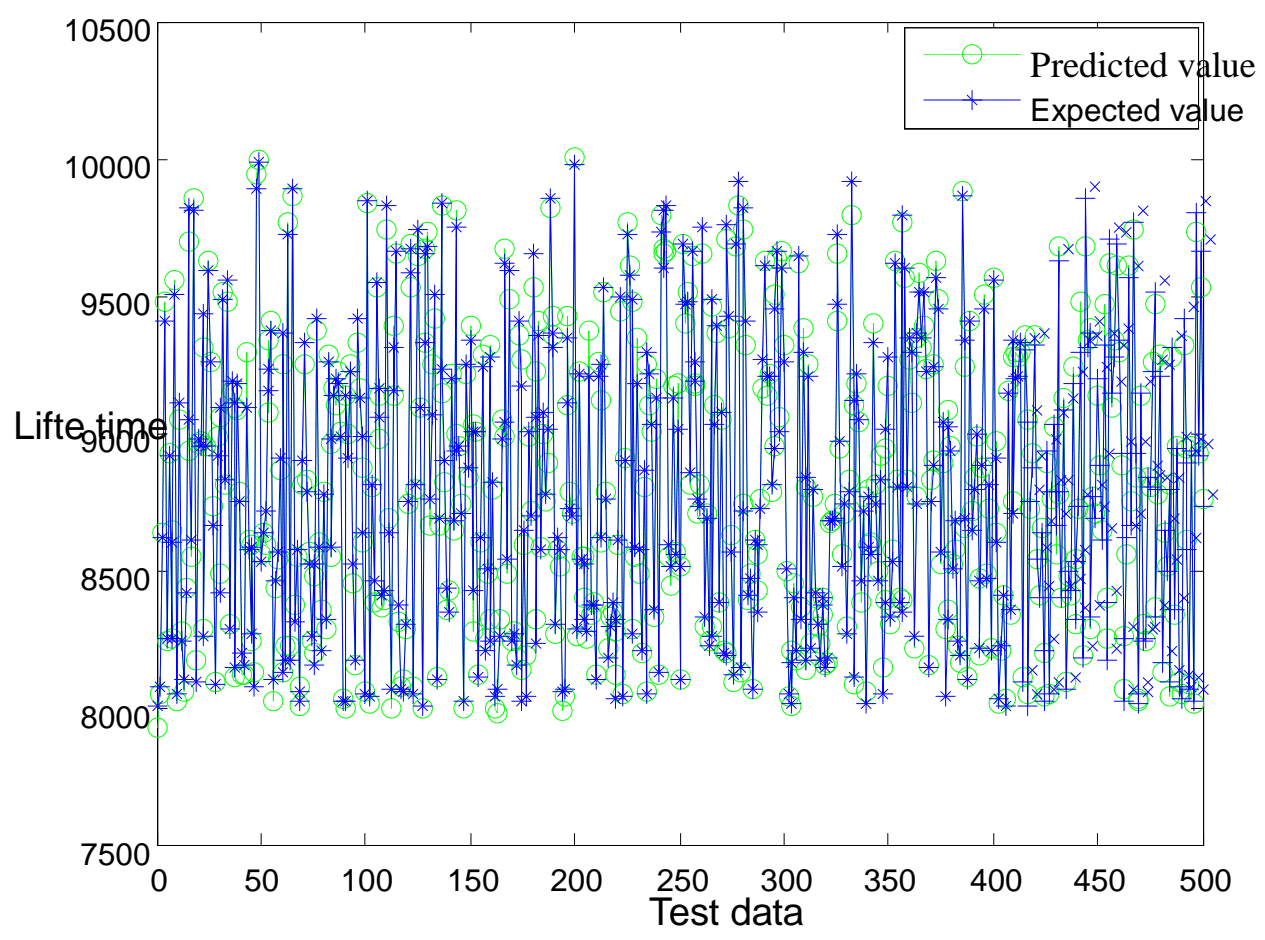

Figure 8. Prediction Output of the Proposed Algorithm 


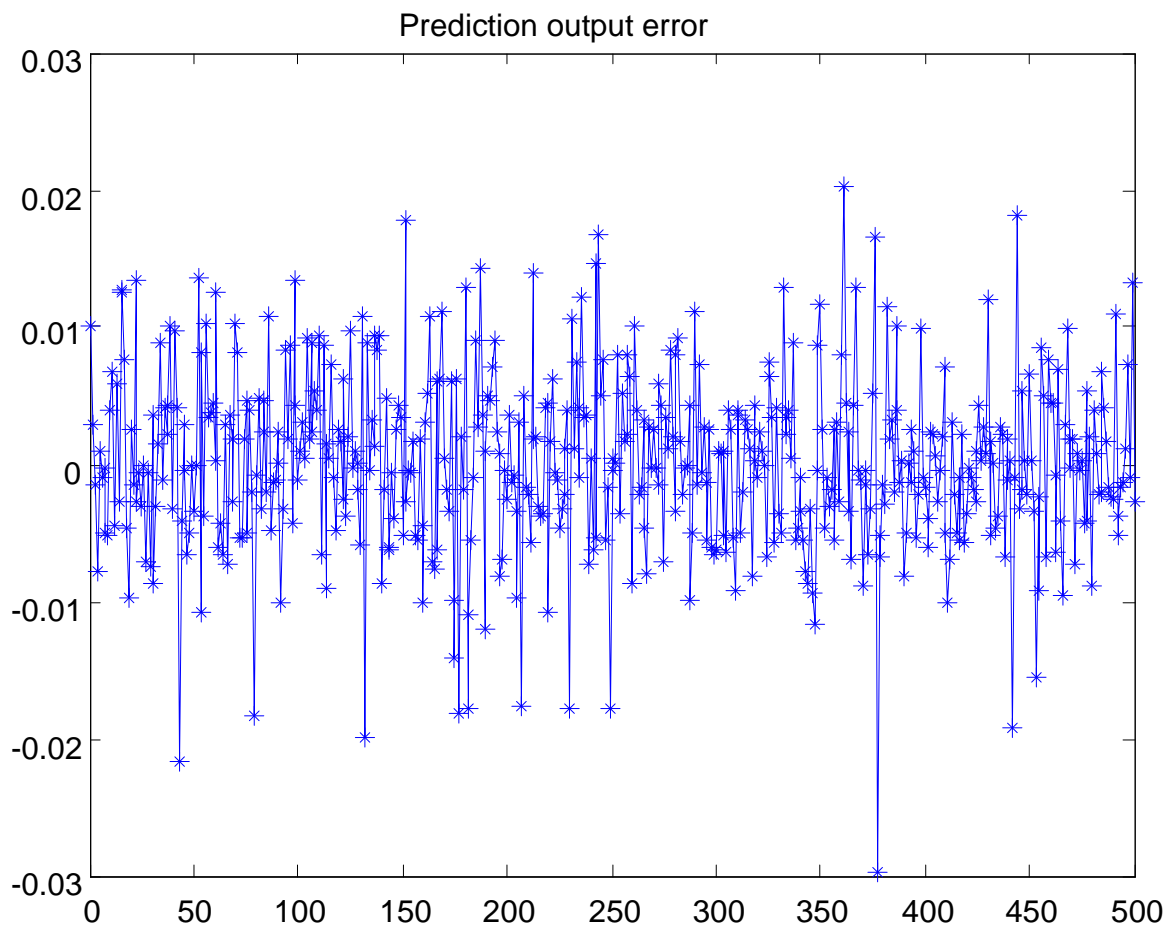

Figure 9. Output Error of the Proposed Algorithm

do so, currently, researchers have discussed this problem by using FT analysis method. However, this method has many defects in analyzing the reliability of mechanical systems, such as, it cannot achieve the dynamic description of reliability and the building process of FT model needs a variety of elements. To deal with this problem, this paper proposes reliability modeling and evaluation issues of electric vehicle motor by using FT based fuzzy Petri nets for the first time. Based on the concepts of FT and fuzzy Petri nets, combined with the defined transformation rules of their elements, a FT based fuzzy Petri nets model for reliability analysis of the mechanical system is obtained. In addition, the reliability calculation method is introduced on FT based fuzzy Petri nets model of the mechanical system and this work designs a hybrid intelligent algorithms integrating fuzzy simulation and NN, namely NN based simulation algorithm, to solve it. Finally, taking an electric vehicle motor as an example, its reliability modeling and evaluation issues are analyzed. The results reveal that they are feasible when used to solve the proposed problems. The obtained results can be used to guide decision makers in making better design when electric vehicle motor is developed and designed.

The future work is to find and use actual reliability test data to validate this method to provide the best decision support for reliability analysis of electric vehicle motor. This work merely analyzes the reliability of mechanical system of the electric vehicle motor. Thus, the reliability issue of integrated system integrating mechanical system and other software needs to be further discussed. In addition, advanced control and technology of electric vehicle motor should be further studied to improve its safety.

\section{Acknowledgements}

This work is financially supported by the Fundamental Research Funds for the Central Universities of China (DL13BB12). 


\section{References}

[1] J. C. González Palencia, T. Furubayashi and T. Nakata, "Energy use and CO2 emissions reduction potential in passenger car fleet using zero emission vehicles and lightweight materials," Energy, vol. 48, no. 1, pp.548-565, 2012.

[2] F. T. Go, A. D. Wahab, M. N. A. Rahman, R. Ramli and H. C. Azhari, "Disassemblability of end-of-life vehicle: a critical review of evaluation methods," Journal of Cleaner Production, vol. 19, no. 13, pp. 1536-1546, 2011.

[3] G. D. Tian, Y. M. Liu, H. Ke and J. W. Chu, "Energy evaluation method and its optimization models for process planning with fuzzy characteristics: A case study in disassembly decision-making," Computers \& Industrial Engineering, vol. 63, no. 3, pp. 553-563, 2012.

[4] G. D. Tian., M. C. Zhou, J. W. Chu and Y. M. Liu, "Probability evaluation models of product disassembly cost subject to random removal time and different removal labor cost," IEEE Transactions Automation Science and Engineering, vol. 9, no.2, pp. 288-295, 2012.

[5] G. D. Tian, J. W. Chu., Y. M. Liu., H. Ke, X. Zhao and G. Xu, "Expected energy analysis for industrial process planning problem with fuzzy time parameters," Computers \& Chemical Engineering, vol. 35, no.12, pp. 2905-2912, 2011.

[6] M. Burgess, N. King, M. Harris and E. Lewis, "Electric vehicle drivers' reported interactions with the public: Driving stereotype change?," Transportation Research Part F: Traffic Psychology and Behaviour, vol. 17, pp. 33-44, 2013.

[7] P. Liu and H. P. Liu, "Application of z-source inverter for permanent-magnet synchronous motor drive system for electric vehicles," Procedia Engineering, vol. 15, pp.309 - 314, 2011.

[8] P. Xu., Z. Hou, G. F. Guo, G. Xu, B. G. Cao and Z. L. Liu, "Driving and control of torque for direct-wheel-driven electric vehicle with motors in serial," Expert Systems with Applications, vol. 38, no. 1, pp.80-86, 2011.

[9] C. Quinn, D. Zimmerle and T. H. Bradley, "The effect of communication architecture on the availability, reliability, and economics of plug-in hybrid electric vehicle-to-grid ancillary services," Journal of Power Sources, vol. 195, no.5, pp. 1500-1509, 2010.

[10] X. H. Zhu, S. M. Cui, N. Shi and Y. L. Min, "Grey prediction model of motor reliability of electric vehicle," Electric Machines and Control, vol. 16, no.8, pp. 42-46, 2012.

[11] X. H. Zhu, S. M. Cui, N. Shi and Y. L. Min, "Grey prediction model of electric vehicle motor based on particle swarm optimization,” High Voltage Engineering, vol. 38, no.6, pp.1391-1396, 2012.

[12] K, Mokhtari., J. Ren, C. Roberts and J. Wang, "Application of a generic bow-tie based risk analysis framework on risk management of sea ports and offshore terminals," Journal of Hazardous Materials, vol. 192, no. 2, pp. 465-475, 2011.

[13] S. R. Cheng, B. S. Lin, B .M. Hsu and M. H. Shu, "Fault-tree analysis for liquefied natural gas terminal emergency shutdown system," Expert Systems with Application, vol. 36, no.9, 11918-11924, 2009.

[14] A. Mentes and I. H. Helvacioglu, "An application of fuzzy fault tree analysis for spread mooring systems," Ocean Engineering, vol. 38, no.2-3, pp. 285-294, 2011.

[15] J. N. Wu, S. Z. Yan and L. Y. Xie, "Reliability analysis method of a solar array by using fault tree analysis and fuzzy reasoning Petri net," Acta Astronautica, vol. 69, no. 11-12, pp.960-968, 2011.

[16] M. C. Zhou and F. Dicesare. Petri net synthesis for discrete event control of manufacturing systems. Kluwer Academic Publishers, 1993.

[17] M. C, Zhou. Modeling, simulation, and control of flexible manufacturing systems: a Petri net approach, World Scientific Publishing Company Incorporated, 1999.

[18] H. S. Hu, M. C. Zhou, Z. W. Li and Y. Tang, "An Optimization Approach to Improved Petri Net Controller Design for Automated Manufacturing Systems," IEEE T. Automation Science and Engineering, vol. 10, no. 3, pp. 772-782, 2013.

[19] Z. W. Li and M .C. Zhou, "Elementary siphons of Petri nets and their application to deadlock prevention in flexible manufacturing systems," IEEE Transactions on Systems, Man and Cybernetics, Part A: Systems and Humans, vol. 34, no. 1, pp. 38-51, 2004.

[20] M. Gao, M. C. Zhou and Y. Tang, "Intelligent Decision Making in Disassembly Process Based on Fuzzy Reasoning Petri Nets," IEEE Trans. Syst., Man, Cybern. B. Cybern., vol. 34, no.5, pp. 2029-2034, 2004.

[21] A. Mhalla, S. Collart Dutilleul, E. Craye and M. Benrejeb, "Estimation of failure probability of milk manufacturing unit by fuzzy fault tree analysis," Journal of Intelligent \& Fuzzy Systems, vol. 26, no. 2, 741-750, 2014.

[22] N. Xie and A. Li, "Modeling and analysis of reconfigurable manufacturing system by extended fuzzy Petri nets," Chinese Journal of Mechanical Engineering, vol. 42, no.12, pp.224-231, 2006.

[23] G. Q. Huang, B. Zhang and C. Z. Wang, "Network attack model based on hierarchical expanded fuzzy Petri net," Computer Engineering, vol. 37, no.22, pp.12-18, 2011.

[24] A. Adamyan and D. He, "System Failure Analysis through Counters of Petri Net Models," Quality and Reliability Engineering International, vol. 20, no.4, pp. 317-335, 2004.

[25] B. Bertsche. Reliability in automotive and mechanical engineering. Berlin Heidelberg: Springer-Verlag, 2008. 
[26] D. X. Liu and S. V. Amari. Fault tree analysis. In: Misra KB, editor. Handbook of Performability Engineering. London limited: Springer-Verlag, 2008.

[27] W. Li, Y. Yao, Z. Ding, Q. Pu, "Efficient Mobile Authentication Scheme Preserving User Privacy for Large-Scale Wireless Networks," Ad Hoc \& Sensor Wireless Networks, vol. 17, no. 3-4, pp. 313-339, 2013.

[28] Z. Ding, J.Liu, J.Wang and Fang Wang, "An Executable Service Composition Code Automatic Creation Tool Based on Petri Net Model,"Computing and Informatics, vol. 32, no.5, pp.968-986, 2013.

[29] L. Li., Z. J. Sun, M. C. Zhou and F. Qiao, "Adaptive dispatching rule for semiconductor wafer fabrication facility," IEEE T. Automation Science and Engineering, vol. 10, no.2, pp. 354-364, 2013.

[30] M. C. Zhou., D. L, Guo and F. Dicesare, "Integration of Petri nets and moment generating function approaches for system,” Journal of Systems Integration, vol. 3, no.1, pp. 43-62, 1993

[31] D. L. Guo., F. Dicesare and M. C. Zhou, "A moment generating function based approach for evaluating extended fuzzy Petri nets," IEEE Transactions on Automatic Control, vol. 38, no. 2, pp.321- 327, 1993.

[32] B, Liu. Uncertainty Theory: An Introduction to its Axiomatic Foundations. Berlin: Springer-Verlag, 2004.

[33] G. D. Tian., M. C. Zhou and J. W, Chu, "A chance constrained programming approach to determine the optimal disassembly sequence," IEEE T. Automation Science and Engineering, vol.10, no. 4, pp.1004-1013, 2013.

[34] G. D. Tian, Y. M. Liu, H. W. Zhang, J. W. Chu and G. Xu, "Chance programming models for time-energy trade-off problem of product disassembly process with multiple fuzzy variables," Advanced Science Letters, vol. 4, no.4-5, pp.1851-1854, 2011.

[35] M. A. Naeem, D. J. Dias., R. Tibrewal., P. C. Chang and M . K. Tiwari, "Production planning optimization for manufacturing and remanufacturing system in fuzzy environment," Journal of Intelligent Manufacturing, vol. 24, no.4, pp. 717-728, 2013.

[36] N. V. Gaitonde and R. S. Karnik, "Minimizing burr size in drilling using artificial neural network (ANN)-particle swarm optimization (PSO) approach," Journal of Intelligent Manufacturing, vol. 23, no.5, pp. 1783-1793, 2012.

[37] G. D. Tian, T. G. Qiang, J. W. Chu, G. Xu, and W.Zhou, "Efficiency optimization for disassembly tools via using NN-GA approach," Mathematical Problems in Engineering, Article ID 173736, 8 pages, 2013.

[38] U. Zuperl and F. Cus, "Optimization of cutting conditions during cutting by using neural networks," Robotics and Computer Integrated Manufacturing, vol. 19, no. 1-2, pp. 189-199, 2003.

[39] V. S. Sharma., S. Dhiman, R. Sehgal and S. K. Sharma, "Estimation of cutting forces and surface roughness for hard turning using neural networks," Journal of Intelligent Manufacturing, vol. 19, no.4, pp. 473-483, 2008.

[40] G. D. Tian, T. G. Qiang and J. W. Chu, "Influence factor analysis and prediction models for component removal time in manufacturing," Proceedings of the Institution of Mechanical Engineers, Part B: Journal of Engineering Manufacture, vol. 227, no. 10, pp. 1533-1540, 2013.

[41] Y. L, Min. Research on life prediction and reliability testing method of the electric vehicle drive motor. Master thesis, Harbin Institute of Technology, 2011.

[42] W. H. Sun, H. J. Gao, O. Kaynak, "Finite frequency $H_{\infty}$ control for vehicle active suspension systems," IEEE Transactions on Control Systems Technology, vol. 19, no. 2, pp. 416-422, 2011.

[43] W. H. Sun,, Z. Zhao, H. J. Gao, "Saturated adaptive robust Control for active suspension systems," IEEE Transactions on Industrial Electronics, vol. 60, no.9, pp. 3889-3896, 2013.

[44] W. H. Sun, H. J. Gao, O. Kaynak, "Adaptive backstepping control for active suspension systems with hard constraints," IEEE Transactions on Mechatronics, vol. 18, no.3, pp. 1072-1079, 2013.

\section{Authors}

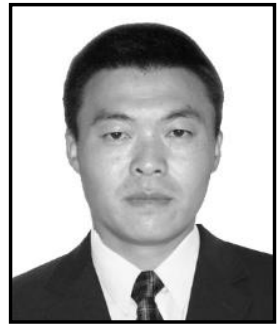

Bing Wang, He received the Bachelor of Science degree in Professional transportation from Northeast Forestry University, Harbin, China, in 2003, the M.S. degree in Dept. of Machinery manufacturing and Automation Specialty. from Harbin University of Science and Technology, Shenyang, China, in 2009. His research focuses on remanufacturing, recycling and reuse of automotive, intelligent optimization algorithm. He has published over 6 journal and conference proceedings papers in the above resea rch areas. 
International Journal of Grid and Distributed Computing

Vol. 9, No. 8 (2016) 